\title{
Inductive extreme non-Arens regularity of the Fourier algebra $A(G)$
}

\author{
by \\ Zhiguo Hu (Windsor, ON)
}

\begin{abstract}
Let $G$ be a non-discrete locally compact group, $A(G)$ the Fourier algebra of $G, \operatorname{VN}(G)$ the von Neumann algebra generated by the left regular representation of $G$ which is identified with $A(G)^{*}$, and $\operatorname{WAP}(\widehat{G})$ the space of all weakly almost periodic functionals on $A(G)$. We show that there exists a directed family $\mathcal{H}$ of open subgroups of $G$ such that: (1) for each $H \in \mathcal{H}, A(H)$ is extremely non-Arens regular; (2) $\operatorname{VN}(G)=\bigcup_{H \in \mathcal{H}} \operatorname{VN}(H)$ and $\operatorname{VN}(G) / \operatorname{WAP}(\widehat{G})=\bigcup_{H \in \mathcal{H}}[\operatorname{VN}(H) / \operatorname{WAP}(\widehat{H})]$; (3) $A(G)=\bigcup_{H \in \mathcal{H}} A(H)$ and it is a WAP-strong inductive union in the sense that the unions in (2) are strongly compatible with it. Furthermore, we prove that the family $\{A(H): H \in \mathcal{H}\}$ of Fourier algebras has a kind of inductively compatible extreme nonArens regularity.
\end{abstract}

1. Introduction. For a Banach algebra $A$, there exist two Banach algebra multiplications on $A^{* *}$ (known as Arens products) which extend the multiplication of $A$ (see Arens [1]). When these two multiplications coincide on $A^{* *}$, the algebra $A$ is said to be Arens regular. Every $C^{*}$-algebra is Arens regular. If $A$ is a commutative Banach algebra, then $A$ is Arens regular if and only if $A^{* *}$ is commutative with respect to either (and hence both) of the Arens products. Let $\mathrm{WAP}(A)$ be the space of all weakly almost periodic functionals on $A$, i.e., $\operatorname{WAP}(A)=\left\{T \in A^{*}:\{u \cdot T: u \in A\right.$ and $\|u\| \leq 1\}$ is relatively weakly compact in $\left.A^{*}\right\}$, where $\langle u \cdot T, v\rangle=\langle T, u v\rangle$ for $v \in A$. It is known that $A$ is Arens regular if and only if $\operatorname{WAP}(A)=A^{*}$ (see Pym [15], and also Duncan and Hosseinium [3]). Hence, the quotient Banach space $A^{*} / \mathrm{WAP}(A)$ measures the non-Arens regularity of $A$ in some sense. In particular, Granirer introduced the concept of "extreme non-Arens regularity". $A$ is called extremely non-Arens regular if $A^{*} / \mathrm{WAP}(A)$ contains a closed linear subspace which has $A^{*}$ as a continuous linear image (see [7]).

2000 Mathematics Subject Classification: 22D25, 43A30, 43A60.

Key words and phrases: locally compact groups, Fourier algebra, weakly almost periodic functionals, Arens regularity.

This research was supported by an NSERC grant. 
Let $G$ be a locally compact group and $A(G)$ the Fourier algebra of $G$. Lau proved that if $G$ is amenable then $A(G)$ is Arens regular if and only if $G$ is finite (see [13, Proposition 3.3]). Generally, Forrest showed that if $A(G)$ is Arens regular then $G$ must be discrete (he even showed this for the Figà-Talamanca Herz algebra $A_{p}(G)$; see [6]). It is still open whether Lau's result is true for non-amenable groups $G$ or for algebras $A_{p}(G)$ with $p \neq 2$. Recently, Granirer investigated the non-Arens regularity of quotients of $A(G)$. A special case of his Corollary 7 in [7] implies that $A(G)$ is extremely non-Arens regular if $G$ is non-discrete and second countable. Let $b(G)$ be the smallest cardinality of an open basis at the unit $e$ of $G$, and $d(G)$ the smallest cardinality of a covering of $G$ by compact sets. It is proved that Granirer's result holds for all non-discrete locally compact groups $G$ satisfying $b(G) \geq d(G)$ (see Hu [10, Corollary 4.2 and Remark 4.7]). In particular, $A(G)$ is extremely non-Arens regular if $G$ is a $\sigma$-compact non-discrete locally compact group.

In this paper we will investigate the non-Arens regularity of $A(G)$ when $b(G)<d(G)$. Let $\operatorname{VN}(G)$ be the von Neumann algebra generated by the left regular representation of $G$. It is well known that $A(G)$ can be identified with the predual of $\operatorname{VN}(G)$, i.e., $\operatorname{VN}(G)=A(G)^{*}$. Let $\operatorname{WAP}(\widehat{G})$ denote the space of all weakly almost periodic functionals on $A(G)$ (i.e., $\operatorname{WAP}(\widehat{G})=$ $\operatorname{WAP}(A(G))$ ). We show (Theorem 5.3) that, for any non-discrete locally compact group $G$ satisfying $b(G)<d(G)$, there exists a directed family $\mathcal{H}$ of open subgroups of $G$ such that:

(1) For each $H \in \mathcal{H}, A(H)$ is extremely non-Arens regular, i.e., for each $H \in \mathcal{H}$, there exists a closed linear subspace $Z_{H}$ of $\operatorname{VN}(H) / \mathrm{WAP}(\widehat{H})$ and a continuous linear map $\Pi_{H}: Z_{H} \rightarrow \mathrm{VN}(H)$ such that $\Pi_{H}\left(Z_{H}\right)=\mathrm{VN}(H)$.

(2) $\operatorname{VN}(G)=\bigcup_{H \in \mathcal{H}} \operatorname{VN}(H)$ is an inductive union of von Neumann algebras and $\operatorname{VN}(G) / \operatorname{WAP}(\widehat{G})=\bigcup_{H \in \mathcal{H}}[\operatorname{VN}(H) / \mathrm{WAP}(\widehat{H})]$ is an inductive union of Banach spaces (see Definition 3.1).

(3) $A(G)=\bigcup_{H \in \mathcal{H}} A(H)$ is an inductive union of Banach algebras and it is a WAP-strong inductive union (see Definition 3.3) in the sense that the two inductive unions in (2) are strongly compatible with the inductive union $A(G)=\bigcup_{H \in \mathcal{H}} A(H)$.

In particular, if $G$ is metrizable, then $H$ is a $\sigma$-compact open subgroup of $G$ for all $H \in \mathcal{H}$, and $A(G)$ is a WAP-strong inductive union of the separable Fourier algebras $\{A(H)\}_{H \in \mathcal{H}}$. Furthermore, we obtain the inductive extreme non-Arens regularity of $A(G)$ by showing that $\left\{\left\|\Pi_{H}\right\|: H \in \mathcal{H}\right\}$ is bounded and the pairs $\left\{Z_{H}, \Pi_{H}\right\}(H \in \mathcal{H})$ are inductively compatible (Theorem 5.10).

The analysis of the relation between open subgroups of $G$ and the support of operators in $\mathrm{VN}(G)$ plays a key role in our discussion of the inductive 
extreme non-Arens regularity of $A(G)$. We show that if $H$ is an open subgroup of a non-discrete locally compact group $G$, then, for any operator $T$ in $\operatorname{VN}(G)$, the support of $T$ can be covered by no more than $b(G)$ cosets of $H$ in $G$ (Proposition 4.1).

Motivated by the inductive limits of $C^{*}$-algebras, in Section 3 we introduce the concept of "inductive union", which provides a natural mechanism to relate the Fourier algebra of a locally compact group to the Fourier algebras of its open subgroups.

2. Preliminaries and notations. Let $G$ be a locally compact group with unit $e$ and a fixed left Haar measure. The Fourier-Stieltjes algebra $B(G)$ is the linear span of positive-definite continuous functions on $G$ and is identified with the Banach dual of the group $C^{*}$-algebra $C^{*}(G)$ of $G$. With the dual norm and the pointwise multiplication, $B(G)$ is a commutative Banach algebra. Let $C_{00}(G)$ be the space of all continuous functions on $G$ with compact support. Then the Fourier algebra $A(G)$ is the closed ideal in $B(G)$ generated by elements in $B(G) \cap C_{00}(G)$. Let $\operatorname{VN}(G)$ be the von Neumann algebra generated by the left regular representation of $G$. Then $A(G)$ can be identified with the predual of $\operatorname{VN}(G)$ (i.e., $\operatorname{VN}(G)=A(G)^{*}$ ) and $\operatorname{VN}(G)$ becomes a $B(G)$-module under the action $\langle u \cdot T, v\rangle=\langle T, u v\rangle$ for $u \in B(G), v \in A(G)$, and $T \in \mathrm{VN}(G)$. Also, $\mathrm{VN}(G)$ coincides with the space of all bounded linear operators on $L^{2}(G)$ which satisfy $T(f * g)=T(f) * g$ for all $f \in L^{2}(G)$ and $g \in C_{00}(G)$. See Eymard [5] for more information on $A(G), B(G)$, and $\operatorname{VN}(G)$.

The space $\{T \in \operatorname{VN}(G): u \mapsto u \cdot T$ is a weakly compact operator from $A(G)$ into $\mathrm{VN}(G)\}$ is called the space of weakly almost periodic functionals on $A(G)$ and is denoted by $\operatorname{WAP}(\widehat{G})$. It turns out that $\operatorname{WAP}(\widehat{G})$ is a selfadjoint closed $B(G)$-submodule of $\operatorname{VN}(G)$. When $G$ is a locally compact abelian group, $\operatorname{WAP}(\widehat{G})$ is identified with the space of weakly almost periodic functions on the dual group of $G$. See Dunkl and Ramirez [4] for more details on $\operatorname{WAP}(\widehat{G})$.

The support of a function $f$ in $L^{2}(G)$ is defined by saying that $x \notin \operatorname{supp} f$ if and only if there exists a neighbourhood $V$ of $x$ such that $\int_{G} f(x) v(x) d x=0$ for all $v \in C_{00}(G)$ with $\operatorname{supp} v \subseteq V$. The support of an operator $T$ in $\operatorname{VN}(G)$ is defined by saying that $x \notin \operatorname{supp} T$ if and only if there exists a neighbourhood $U$ of $e$ such that $x \notin \operatorname{supp}(T u)$ for all $u \in C_{00}(G)$ with supp $u \subseteq U$. An equivalent description for $\operatorname{supp} T$ is that $x \in \operatorname{supp} T$ if and only if $u \cdot T=0$ implies $u(x)=0$ for all $u \in A(G)$ (see Eymard [5] and Herz [8]).

Let $b(G)$ be the smallest cardinality of an open basis at $e$ and $d(G)$ denote the smallest cardinality of a covering of $G$ by compact sets. It is known that $b(G)=d(\widehat{G})$ when $G$ is abelian with dual group $\widehat{G}$ (see Hewitt and Ross $[12,(24.48)])$. Clearly, $G$ is metrizable if and only if $b(G) \leq \aleph_{0}$. 
3. Inductive unions. Inspired by the inductive limits of $C^{*}$-algebras, we introduce the concept of "inductive union", which is of importance for our investigation on the non-Arens regularity of the Fourier algebra $A(G)$.

Definition 3.1. Let $A$ be a Banach space (Banach algebra, $C^{*}$-algebra, respectively) and let $\left\{A_{i}\right\}_{i \in I}$ be a family of Banach spaces (Banach algebras, $C^{*}$-algebras, respectively) indexed by a directed set $I$. We say that $A$ is an inductive union of $\left\{A_{i}\right\}_{i \in I}$ (denoted by $A=\bigsqcup_{i \in I} A_{i}$ ) if there exists a linear isometry (isometric isomorphism, *-isomorphism, respectively) $\Lambda_{i}: A_{i} \rightarrow A$ for each $i \in I$ such that $\Lambda_{i}\left(A_{i}\right) \subseteq \Lambda_{j}\left(A_{j}\right)$ for all $i, j \in I$ with $i \preceq j$ and $A=\bigcup_{i \in I} \Lambda_{i}\left(A_{i}\right)$.

Immediately, we can show the existence of maps $\Lambda_{i j}(i \preceq j)$ compatible with $\left\{\Lambda_{i}\right\}_{i \in I}$.

Corollary 3.2. Let $A=\bigsqcup_{i \in I} A_{i}$ be an inductive union of the family $\left\{A_{i}\right\}_{i \in I}$ of Banach spaces (Banach algebras, $C^{*}$-algebras, respectively) via the linear isometries (isometric isomorphisms, *-isomorphisms, respectively) $\left\{\Lambda_{i}\right\}_{i \in I}$. Then, for all $i, j \in I$ with $i \preceq j$, there exists a unique linear isometry (isometric isomorphism, *-isomorphism, respectively) $\Lambda_{i j}: A_{i}$ $\rightarrow A_{j}$ such that:

(a) $\Lambda_{j} \Lambda_{i j}=\Lambda_{i}$ for all $i, j \in I$ with $i \preceq j$.

(b) $\Lambda_{j k} \Lambda_{i j}=\Lambda_{i k}$ if $i, j, k \in I$ and $i \preceq j \preceq k$.

Proof. Let $i, j \in I$ and $i \preceq j$. Note that $\Lambda_{i}\left(A_{i}\right) \subseteq \Lambda_{j}\left(A_{j}\right)$ and hence $\Lambda_{i}\left(A_{i}\right)$ is a closed linear subspace (subalgebra, $C^{*}$-subalgebra, respectively) of $\Lambda_{j}\left(A_{j}\right)$. Define $\Lambda_{i j}=\left.\left(\Lambda_{j}\right)^{-1}\right|_{\Lambda_{i}\left(A_{i}\right)} \Lambda_{i}$. Then $\Lambda_{i j}: A_{i} \rightarrow A_{j}$ is a linear isometry (isometric isomorphism, $*$-isomorphism, respectively). By the definition of $\Lambda_{i j}$, it can be seen that (a) holds and the map $\Lambda_{i j}$ satisfying (a) is unique.

Suppose that $i, j, k \in I$ and $i \preceq j \preceq k$. By (a), we have $\Lambda_{k}\left(\Lambda_{j k} \Lambda_{i j}\right)=$ $\Lambda_{j} \Lambda_{i j}=\Lambda_{i}=\Lambda_{k} \Lambda_{i k}$, i.e., $\Lambda_{j k} \Lambda_{i j}=\Lambda_{i k}$ since $\Lambda_{k}$ is one-to-one. Therefore, (b) is true.

When $A$ is an inductive union of $\left\{A_{i}\right\}_{i \in I}$, it is interesting to know if $A^{*}$ is an inductive union of $\left\{A_{i}^{*}\right\}_{i \in I}$ and if a quotient space of $A^{*}$ is an inductive union of the corresponding quotient spaces of $A_{i}^{*}(i \in I)$, etc. For our purpose, we only consider the following "WAP" strongly compatible inductive unions of Banach algebras. Recall that, for a Banach algebra $A$, $\mathrm{WAP}(A)$ denotes the space of all weakly almost periodic functionals on $A$.

Definition 3.3. Let $A$ be a Banach algebra and let $A=\bigsqcup_{i \in I} A_{i}$ be an inductive union of the Banach algebras $\left\{A_{i}\right\}_{i \in I}$ via the isometric isomor- 
phisms $\left\{\Lambda_{i}\right\}_{i \in I}$. We say that $A$ is a WAP-strong inductive union of $\left\{A_{i}\right\}_{i \in I}$ if the following hold.

(1) $A^{*}=\bigsqcup_{i \in I} A_{i}^{*}$ is an inductive union of the Banach spaces $\left\{A_{i}^{*}\right\}_{i \in I}$ via some linear isometries $\left\{\Phi_{i}\right\}_{i \in I}$ such that, for all $i \in I, \Lambda_{i}^{*} \Phi_{i}=\operatorname{Id}$ and $\Phi_{i}(u \cdot T)=\Lambda_{i}(u) \cdot \Phi_{i}(T)$ for $u \in A_{i}$ and $T \in A_{i}^{*}$.

(2) For all $i \in I, \Phi_{i}\left(\operatorname{WAP}\left(A_{i}\right)\right)=\operatorname{WAP}(A) \cap \Phi_{i}\left(A_{i}^{*}\right)$ and $\Phi_{i}$ lifts a linear isometry $\Gamma_{i}: A_{i}^{*} / \mathrm{WAP}\left(A_{i}\right) \rightarrow A^{*} / \mathrm{WAP}(A)$.

It is easy to see that (1) and (2) in Definition 3.3 are equivalent to the following two conditions.

Corollary 3.4. Let $A=\bigsqcup_{i \in I} A_{i}$ be an inductive union of the Banach algebras $\left\{A_{i}\right\}_{i \in I}$ via $\left\{\Lambda_{i}\right\}_{i \in I}$. Then $A$ is a WAP-strong inductive union of $\left\{A_{i}\right\}_{i \in I}$ if and only if the following conditions are satisfied:

$(1)^{\prime} A^{*}=\bigsqcup_{i \in I} A_{i}^{*}$ is an inductive union of $\left\{A_{i}^{*}\right\}_{i \in I}$ via $\left\{\Phi_{i}\right\}_{i \in I}$ such that, for all $i \in I, \Phi_{i} \Lambda_{i}^{*}: A^{*} \rightarrow A^{*}$ is a $\Lambda_{i}\left(A_{i}\right)$-invariant projection (i.e., $\left(\Phi_{i} \Lambda_{i}^{*}\right)^{2}=\Phi_{i} \Lambda_{i}^{*}$ and $\Phi_{i} \Lambda_{i}^{*}(v \cdot T)=v \cdot\left[\Phi_{i} \Lambda_{i}^{*}(T)\right]$ for all $v \in \Lambda_{i}\left(A_{i}\right)$ and $\left.T \in A^{*}\right)$.

$(2)^{\prime} \operatorname{WAP}(A)=\bigsqcup_{i \in I} \operatorname{WAP}\left(A_{i}\right)$ is an inductive union of the Banach spaces $\left\{\operatorname{WAP}\left(A_{i}\right)\right\}_{i \in I}$ via the restrictions $\left\{\left.\Phi_{i}\right|_{\mathrm{WAP}\left(A_{i}\right)}\right\}_{i \in I}$ and $A^{*} / \operatorname{WAP}(A)$ $=\bigsqcup_{i \in I}\left[A_{i}^{*} / \mathrm{WAP}\left(A_{i}\right)\right]$ is an inductive union of the quotient Banach spaces $\left\{A_{i}^{*} / \operatorname{WAP}\left(A_{i}\right)\right\}_{i \in I}$ via $\left\{\Gamma_{i}\right\}_{i \in I}$ such that $\Gamma_{i} \varrho_{i}=\varrho \Phi_{i}$ for all $i \in I$, where $\varrho_{i}: A_{i}^{*} \rightarrow A_{i}^{*} / \mathrm{WAP}\left(A_{i}\right)$ and $\varrho: A^{*} \rightarrow A^{*} / \mathrm{WAP}(A)$ are the canonical quotient maps.

Analogously to Corollary 3.2, we are able to get maps $\Phi_{i j}$ and $\Gamma_{i j}(i \preceq j)$ which are compatible with $\left\{\Phi_{i}\right\}_{i \in I}$ and $\left\{\Gamma_{i}\right\}_{i \in I}$, respectively.

Corollary 3.5. Let $A=\bigsqcup_{i \in I} A_{i}$ be a WAP-strong inductive union of the Banach algebras $\left\{A_{i}\right\}_{i \in I}$ via the maps $\left\{\Lambda_{i}\right\}_{i \in I}$ with $A^{*}=\bigsqcup_{i \in I} A_{i}^{*}$ via $\left\{\Phi_{i}\right\}_{i \in I}$ and $A^{*} / \operatorname{WAP}(A)=\bigsqcup_{i \in I}\left[A_{i}^{*} / \operatorname{WAP}\left(A_{i}\right)\right]$ via $\left\{\Gamma_{i}\right\}_{i \in I}$. Then, for all $i, j \in I$ with $i \preceq j$, there exist unique linear isometries $\Phi_{i j}: A_{i}^{*} \rightarrow A_{j}^{*}$ and $\Gamma_{i j}: A_{i}^{*} / \mathrm{WAP}\left(A_{i}\right) \rightarrow A_{j}^{*} / \mathrm{WAP}\left(A_{j}\right)$ such that the following hold:

(a) $\Phi_{j} \Phi_{i j}=\Phi_{i}$ and $\Gamma_{j} \Gamma_{i j}=\Gamma_{i}$ for all $i, j \in I$ with $i \preceq j$.

(b) $\Phi_{j k} \Phi_{i j}=\Phi_{i k}$ and $\Gamma_{j k} \Gamma_{i j}=\Gamma_{i k}$ if $i, j, k \in I$ and $i \preceq j \preceq k$.

(c) $\Lambda_{i j}^{*} \Phi_{i j}=\operatorname{Id}$ and $\Phi_{i j}(u \cdot T)=\Lambda_{i j}(u) \cdot \Phi_{i j}(T)$ for all $i, j \in I$ with $i \preceq j, u \in A_{i}$ and $T \in A_{i}^{*}$, where $\Lambda_{i j}: A_{i} \rightarrow A_{j}$ is the same map as in Corollary 3.2.

(d) $\Phi_{i j}\left(\operatorname{WAP}\left(A_{i}\right)\right)=\operatorname{WAP}\left(A_{j}\right) \cap \Phi_{i j}\left(A_{i}^{*}\right)$ and $\Gamma_{i j} \varrho_{i}=\varrho_{j} \Phi_{i j}$ if $i, j \in I$ and $i \preceq j$ (i.e., $\Gamma_{i j}$ is the map lifted by $\Phi_{i j}$ ).

Proof. It can be seen that (a) and (b) hold by the same argument as in the proof of Corollary 3.2. Clearly, the maps $\Phi_{i j}$ and $\Gamma_{i j}$ satisfying (a) are unique. 
Let $i, j \in I$ and $i \preceq j$. Note that $\Lambda_{i}^{*} \Phi_{i}=\mathrm{Id}, \Lambda_{i}^{*}=\Lambda_{i j}^{*} \Lambda_{j}^{*}$ (by Corollary 3.2), and $\Phi_{i}=\Phi_{j} \Phi_{i j}$. Therefore, $\Lambda_{i j}^{*} \Phi_{i j}=\Lambda_{i j}^{*}\left(\Lambda_{j}^{*} \Phi_{j}\right) \Phi_{i j}=$ $\left(\Lambda_{i j}^{*} \Lambda_{j}^{*}\right)\left(\Phi_{j} \Phi_{i j}\right)=\Lambda_{i}^{*} \Phi_{i}=\mathrm{Id}$, i.e., $\Lambda_{i j}^{*} \Phi_{i j}=\mathrm{Id}$. Suppose that $u \in A_{i}$ and $T \in A_{i}^{*}$. Then

$$
\begin{aligned}
\Phi_{j}\left[\Phi_{i j}(u \cdot T)\right] & =\Phi_{i}(u \cdot T)=\Lambda_{i}(u) \cdot \Phi_{i}(T) \\
& =\Lambda_{j}\left[\Lambda_{i j}(u)\right] \cdot \Phi_{j}\left[\Phi_{i j}(T)\right]=\Phi_{j}\left[\Lambda_{i j}(u) \cdot \Phi_{i j}(T)\right] .
\end{aligned}
$$

We conclude that $\Phi_{i j}(u \cdot T)=\Lambda_{i j}(u) \cdot \Phi_{i j}(T)$ since the map $\Phi_{j}$ is one-to-one. Therefore, (c) is true.

Note that $\Phi_{i}\left(\operatorname{WAP}\left(A_{i}\right)\right) \subseteq \Phi_{j}\left(\operatorname{WAP}\left(A_{j}\right)\right) \subseteq \operatorname{WAP}(A)$ and hence we have $\Phi_{i}\left(\operatorname{WAP}\left(A_{i}\right)\right)=\Phi_{j}\left(\operatorname{WAP}\left(A_{j}\right)\right) \cap \Phi_{i}\left(A_{i}^{*}\right)$, that is, $\Phi_{j}\left[\Phi_{i j}\left(\operatorname{WAP}\left(A_{i}\right)\right)\right]=$ $\Phi_{j}\left[\operatorname{WAP}\left(A_{j}\right) \cap \Phi_{i j}\left(A_{i}^{*}\right)\right]$. Therefore, $\Phi_{i j}\left(\operatorname{WAP}\left(A_{i}\right)\right)=\operatorname{WAP}\left(A_{j}\right) \cap \Phi_{i j}\left(A_{i}^{*}\right)$. Finally, by using the facts that $\Gamma_{j} \Gamma_{i j}=\Gamma_{i}, \Gamma_{i} \varrho_{i}=\varrho \Phi_{i}$, and $\Phi_{i}=\Phi_{j} \Phi_{i j}$, we have $\Gamma_{j}\left(\Gamma_{i j} \varrho_{i}\right)=\Gamma_{i} \varrho_{i}=\varrho \Phi_{i}=\varrho \Phi_{j} \Phi_{i j}=\Gamma_{j}\left(\varrho_{j} \Phi_{i j}\right)$. It follows that $\Gamma_{i j} \varrho_{i}=\varrho_{j} \Phi_{i j}$ since $\Gamma_{j}$ is one-to-one. Therefore, (d) holds.

4. Open subgroups, support of $T$ in $\operatorname{VN}(G)$, and isometric embeddings. In this section, $G$ is a locally compact group and $H$ is an open subgroup of $G$. Let $\operatorname{VN}_{H}(G)$ denote the von Neumann subalgebra of $\operatorname{VN}(G)$ generated by $\left\{\lambda_{G}(x): x \in H\right\}$, where $\lambda_{G}$ is the left regular representation of $G$. Then $\operatorname{VN}_{H}(G)=\{T \in \mathrm{VN}(G): \operatorname{supp} T \subseteq H\}$ (see Chou [2, Lemma 4.2]). Let $1_{H} \in B(G)$ be the characteristic function of $H$. Then $1_{H} \cdot T \in \mathrm{VN}_{H}(G)$ for all $T \in \mathrm{VN}(G)$ and $T=1_{H} \cdot T$ if $T \in \mathrm{VN}_{H}(G)$. Therefore, $\mathrm{VN}_{H}(G)=1_{H} \cdot \mathrm{VN}(G)$.

It is known that if an element $T$ of $\operatorname{VN}(G)$ is the left convolution operator by a bounded complex-valued regular Borel measure $\mu$ on $G$, then the support of $T$ is just the support of the measure $\mu$ and hence it is a countable union of compact sets in $G$ by the regularity of $\mu$.

Generally, for an arbitrary operator $T$ in $\operatorname{VN}(G)$, we are concerned with the question of how many cosets $g H$ we will need at least to cover the support of $T$. If $G$ is discrete, then every element $T$ of $\operatorname{VN}(G)$ is identified with a left convolution operator by a function in $l^{2}(G)$ and so the support of $T$ is a countable subset of $G$. In the following, we will consider the case when $G$ is non-discrete.

Proposition 4.1. Let $G$ be a non-discrete locally compact group and let $H$ be an open subgroup of $G$. Then, for any $T \in \mathrm{VN}(G)$, there are at most $b(G)$ cosets $g H(g \in G)$ such that $\operatorname{supp} T \cap g H \neq \emptyset$.

Proof. Replacing $H$ by a $\sigma$-compact open subgroup of $H$, we may assume that $H$ is a $\sigma$-compact open subgroup of $G$.

Let $\mathcal{U}$ be a compact neighbourhood system at $e$ such that $\operatorname{card}(\mathcal{U})=$ $b(G)$. Then $\mathcal{U}$ is a directed set under the relation $U \preceq V$ if and only if 
$V \subseteq U$. For each $U \in \mathcal{U}$, let $h_{U}=(1 /|U|) 1_{U}$ and $T_{U}=T\left(h_{U}\right) \in L^{2}(G)$, where $|U|$ is the left Haar measure of $U$ and $1_{U}$ denotes the characteristic function of $U$. By $[12,(20.15)]$, for all $f \in L^{2}(G), \lim _{U}\left\|h_{U} * f-f\right\|_{2}=0$. If $f \in C_{00}(G)$, then $T\left(h_{U} * f\right)=T\left(h_{U}\right) * f=T_{U} * f$ and hence $T(f)=$ $\lim _{U}\left(T_{U} * f\right)$ in the $\|\cdot\|_{2}$-norm. Therefore, $T$ is completely determined by the net $\left(T_{U}\right)_{U \in \mathcal{U}}$ in $L^{2}(G)$ since $C_{00}(G)$ is $\|\cdot\|_{2}$-norm dense in $L^{2}(G)$. For each $U \in \mathcal{U}$, since $T_{U} \in L^{2}(G)$, there exists a sequence $\left\{g_{U}^{n}\right\}_{n}$ in $G$ such that $\operatorname{supp} T_{U} \subseteq \bigcup_{n=1}^{\infty} g_{U}^{n} H$.

Fix a compact neighbourhood $V$ of $e$. Since $H$ is $\sigma$-compact, $H V$ and hence $\bigcup_{n=1}^{\infty} g_{U}^{n} H V$ is a countable union of compact sets. Therefore, $\bigcup_{n=1}^{\infty} g_{U}^{n} H V$ can be covered by countably many cosets $g H$. Note that $\operatorname{card}(\mathcal{U})$ $=b(G) \geq \aleph_{0}$. It follows that there exists a subset $B$ of $G$ such that $\operatorname{card}(B) \leq b(G)=\operatorname{card}(\mathcal{U})$ and $\bigcup_{U \in \mathcal{U}} \bigcup_{n=1}^{\infty} g_{U}^{n} H V \subseteq \bigcup_{g \in B} g H$.

To complete the proof, we only need to show that $\operatorname{supp} T \subseteq \bigcup_{g \in B} g H$.

Suppose $x \in G \backslash \bigcup_{g \in B} g H$. In the following, we will prove that $x \notin$ $\operatorname{supp} T(f)$ for all $f \in C_{00}(G)$ with $\operatorname{supp} f \subseteq V$ and it follows that $x \notin \operatorname{supp} T$.

Let $f \in C_{00}(G)$ and $\operatorname{supp} f \subseteq V$. Then $T(f)=\lim _{U \in \mathcal{U}}\left(T_{U} * f\right)$ in the $\|\cdot\|_{2}$-norm. Recall that, for each $U \in \mathcal{U}$, supp $T_{U} \subseteq \bigcup_{n=1}^{\infty} g_{U}^{n} H$ and hence $\operatorname{supp}\left(T_{U} * f\right) \subseteq \bigcup_{n=1}^{\infty} g_{U}^{n} H V \subseteq \bigcup_{g \in B} g H$. Also note that $\bigcup_{g \in B} g H$ is closed in $G$. Therefore, $\operatorname{supp} T(f) \subseteq \bigcup_{g \in B} g H$ and we have $x \notin \operatorname{supp} T(f)$.

Corollary 4.2. Let $G$ be a metrizable locally compact group and let $H$ be an open subgroup of $G$. Then, for any $T \in \mathrm{VN}(G)$, there exists a sequence $\left\{g_{n}\right\}_{n}$ in $G$ such that $\operatorname{supp} T \subseteq \bigcup_{n=1}^{\infty} g_{n} H$.

REMARK 4.3. Let $G$ be a locally compact group and let $H$ be an open subgroup of $G$. If $T \in \overline{\operatorname{span}}\left[\lambda_{G}(G) \mathrm{VN}_{H}(G)\right]$ (the norm closed linear span generated by the translates of elements in $\operatorname{VN}_{H}(G)$ ), then the support of $T$ can be covered by countably many cosets $g H$. However, it is possible that the support of any operator in $\operatorname{VN}(G)$ can be covered by countably many cosets $g H$ (e.g., when $G$ is metrizable or $\sigma$-compact) but $\operatorname{VN}(G) \neq$ $\overline{\operatorname{span}}\left[\lambda_{G}(G) \mathrm{VN}_{H}(G)\right]$. For example, let $G$ be a non-compact metrizable locally compact group containing a compact open subgroup $H$. Then $\operatorname{VN}(H)=$ $U C(\widehat{H})$ (the $C^{*}$-algebra of uniformly continuous functionals on $A(H)$ introduced by Granirer) and thus $\overline{\operatorname{span}}\left[\lambda_{G}(G) \mathrm{VN}_{H}(G)\right]=\mathrm{UC}(\widehat{G})$ (see Hu [11, Proposition 3.5]). Now $\overline{\operatorname{span}}\left[\lambda_{G}(G) \mathrm{VN}_{H}(G)\right]=\mathrm{UC}(\widehat{G}) \varsubsetneqq \mathrm{VN}(G)$ because $G$ is non-compact.

Corollary 4.4. Let $G$ be a metrizable locally compact group. Then, for any $T \in \operatorname{VN}(G)$, there exists a $\sigma$-compact open subgroup $H$ of $G$ such that $\operatorname{supp} T \subseteq H$.

Proof. Let $G_{0}$ be a $\sigma$-compact open subgroup of $G$. Let $T \in \operatorname{VN}(G)$. By Corollary 4.2, there exists a sequence $\left\{g_{n}\right\}_{n}$ in $G$ such that $\operatorname{supp} T \subseteq$ 
$\bigcup_{n=1}^{\infty} g_{n} G_{0}$. Let $H$ be the open subgroup of $G$ generated by $G_{0} \cup \bigcup_{n=1}^{\infty} g_{n} G_{0}$. Then $H$ is a $\sigma$-compact open subgroup of $G$ and $\operatorname{supp} T \subseteq H$.

Let $r: A(G) \rightarrow A(H)$ be the restriction map. According to Eymard [5], $r$ is a linear contractive surjection and its adjoint $r^{*}$ is a $*$-isomorphism of the von Neumann algebra $\mathrm{VN}(H)$ onto the von Neumann subalgebra $\mathrm{VN}_{H}(G)$ of $\operatorname{VN}(G)$ (see [5, (3.21)], where $r^{*}(T)$ is denoted as $T^{\circ}$ for $T \in \mathrm{VN}(H)$ ). It is known that $r^{*}(\operatorname{WAP}(\widehat{H}))=\operatorname{WAP}(\widehat{G}) \cap \mathrm{VN}_{H}(G)$ (see Chou [2, Lemma 4.2]). Therefore, the $*$-isomorphism $r^{*}$ lifts a linear map from the quotient Banach space $\operatorname{VN}(H) / \operatorname{WAP}(\widehat{H})$ into the quotient Banach space $\operatorname{VN}(G) / \operatorname{WAP}(\widehat{G})$. Let $\operatorname{VN}_{H}(G) / \operatorname{WAP}(\widehat{G})$ denote the linear subspace $\{T+\operatorname{WAP}(\widehat{G}): T \in$ $\left.\operatorname{VN}_{H}(G)\right\}$ of $\operatorname{VN}(G) / \operatorname{WAP}(\widehat{G})$. In the following we will show that in fact $r^{*}$ lifts a linear isometry between $\operatorname{VN}(H) / \mathrm{WAP}(\widehat{H})$ and $\operatorname{VN}_{H}(G) / \mathrm{WAP}(\widehat{G})$.

Proposition 4.5. For $T \in \operatorname{VN}(H)$, define $\widetilde{r}^{*}(T+\operatorname{WAP}(\widehat{H}))=r^{*}(T)+$ $\operatorname{WAP}(\widehat{G})$. Then $\widetilde{r}^{*}: \operatorname{VN}(H) / \operatorname{WAP}(\widehat{H}) \rightarrow \operatorname{VN}(G) / \operatorname{WAP}(\widehat{G})$ is a linear isometry with range $\operatorname{VN}_{H}(G) / \mathrm{WAP}(\widehat{G})$ and the following diagram commutes:

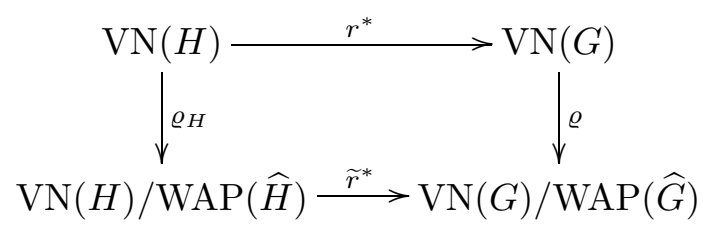

where $\varrho_{H}$ and $\varrho$ are the canonical quotient maps.

Proof. Since $r^{*}(\mathrm{VN}(H))=\operatorname{VN}_{H}(G)$ and $r^{*}(\operatorname{WAP}(\widehat{H}))=\operatorname{WAP}(\widehat{G}) \cap$ $\mathrm{VN}_{H}(G)$, by the definition, $\widetilde{r}^{*}: \operatorname{VN}(H) / \operatorname{WAP}(\widehat{H}) \rightarrow \operatorname{VN}(G) / \operatorname{WAP}(\widehat{G})$ is well defined, linear, and onto the linear subspace $\operatorname{VN}_{H}(G) / \operatorname{WAP}(\widehat{G})$ of $\operatorname{VN}(G) / \operatorname{WAP}(\widehat{G})$. According to the definition of $\widetilde{r}^{*}$, it is clear that the diagram is commutative. To complete the proof, we only need to show that $\widetilde{r}^{*}$ is an isometry.

Let $T \in \mathrm{VN}(H)$. Obviously, $\left\|\widetilde{r}^{*}(T+\mathrm{WAP}(\widehat{H}))\right\| \leq\|T+\mathrm{WAP}(\widehat{H})\|$ since $\left\|\widetilde{r}^{*}\right\| \leq\left\|r^{*}\right\|=1$. Conversely, let $W \in \operatorname{WAP}(\widehat{G})$. Then $W=W_{1}+W_{2}$, where $W_{1}=1_{H} \cdot W$ and hence $W_{1} \in \operatorname{WAP}(\widehat{G}) \cap \mathrm{VN}_{H}(G)$, and $W_{2}=W-W_{1} \in$ $\operatorname{WAP}(\widehat{G})$ with supp $W_{2} \subseteq G \backslash H$. Thus, $W_{1}=r^{*}\left(V_{1}\right)$ for some $V_{1} \in \operatorname{WAP}(\widehat{H})$. So,

$$
\begin{aligned}
\left\|r^{*}(T)+W\right\| & =\left\|r^{*}(T)+r^{*}\left(V_{1}\right)+W_{2}\right\| \\
& \geq\left\|1_{H} \cdot\left(r^{*}(T)+r^{*}\left(V_{1}\right)+W_{2}\right)\right\| \\
& =\left\|r^{*}(T)+r^{*}\left(V_{1}\right)\right\| \quad\left(\text { since } 1_{H} \cdot W_{2}=0\right) \\
& =\left\|T+V_{1}\right\| \\
& \geq\|T+\operatorname{WAP}(\widehat{H})\| .
\end{aligned}
$$


Since $W \in \operatorname{WAP}(\widehat{G})$ is arbitrary, it follows that

$$
\left\|r^{*}(T)+\operatorname{WAP}(\widehat{G})\right\| \geq\|T+\operatorname{WAP}(\widehat{H})\|,
$$

i.e., $\left\|\widetilde{r}^{*}(T+\operatorname{WAP}(\widehat{H}))\right\| \geq\|T+\operatorname{WAP}(\widehat{H})\|$. Therefore, $\widetilde{r}^{*}$ is a linear isometry.

Remark 4.6. Let $V$ be any closed $B(G)$-submodule of $\operatorname{VN}(G)$ and let $V_{H}=\left(r^{*}\right)^{-1}\left[V \cap \mathrm{VN}_{H}(G)\right]$. Then $V_{H}$ is a closed $B(H)$-submodule of $\mathrm{VN}(H)$ and $r^{*}\left(V_{H}\right)=V \cap \mathrm{VN}_{H}(G)$. From the proof it can be seen that Proposition 4.5 holds if $\operatorname{WAP}(\widehat{G})$ and $\operatorname{WAP}(\widehat{H})$ are replaced by $V$ and $V_{H}$, respectively. In particular, if we take $V=\operatorname{AP}(\widehat{G}), \operatorname{UC}(\widehat{G}), C_{\mathrm{r}}^{*}(G)$, and $C_{\delta}^{*}(G)$ (the space of almost periodic functionals on $A(G)$, the space of uniformly continuous functionals on $A(G)$, the reduced group $C^{*}$-algebra of $G$, and the $C^{*}$-algebra generated by $\left\{\lambda_{G}(x): x \in G\right\}$, respectively), then we will get $V_{H}=\mathrm{AP}(\widehat{H}), \mathrm{UC}(\widehat{H}), C_{\mathrm{r}}^{*}(H)$, and $C_{\delta}^{*}(H)$, respectively (cf. [11]).

5. Inductive extreme non-Arens regularity of $A(G)$. Throughout this section, we assume that $G$ is a non-discrete locally compact group and $G_{0}$ is a $\sigma$-compact open subgroup of $G$.

Let $T \in \operatorname{VN}(G)$. By Proposition 4.1, there exists a subset $B$ of $G$ such that $\operatorname{card}(B) \leq b(G)$ and $\operatorname{supp} T \cap g G_{0}=\emptyset$ for all $g \in G \backslash B$. Hence, $\operatorname{supp} T \subseteq \bigcup_{g \in B} g G_{0}$. Let $H_{B}$ be the open subgroup of $G$ generated by $G_{0} \cup \cup_{g \in B} g G_{0}$, i.e.,

$$
H_{B}=\bigcup_{n=1}^{\infty}\left\{\left[G_{0} \cup \bigcup_{g \in B} g G_{0}\right] \cup\left[G_{0} \cup \bigcup_{g \in B} g G_{0}\right]^{-1}\right\}^{n} .
$$

Then we have $T \in V N_{H_{B}}(G)$ and $H_{B}$ can be covered by no more than $b(G)$ compact sets (since $G_{0}$ is $\sigma$-compact and $b(G) \geq \aleph_{0}$ ). Therefore, $d\left(H_{B}\right) \leq$ $b\left(H_{B}\right)(=b(G))$. According to the result of $\mathrm{Hu}[10$, Corollary 4.2 and Remark 4.7], $A\left(H_{B}\right)$ is extremely non-Arens regular.

To obtain the inductive extreme non-Arens regularity of $A(G)$, we need to consider the following maps.

DeFinition 5.1. Let $H$ and $J$ be open subgroups of $G$ and $H \subseteq J$. The maps $\Lambda_{H J}: A(H) \rightarrow A(J), \Phi_{H J}: \operatorname{VN}(H) \rightarrow V N(J)$, and $\Gamma_{H J}:$ $\mathrm{VN}(H) / \mathrm{WAP}(\widehat{H}) \rightarrow \operatorname{VN}(J) / \mathrm{WAP}(\widehat{J})$ are defined as follows: for $u \in A(H)$ and $T \in \mathrm{VN}(H)$,

$$
\begin{aligned}
\Lambda_{H J}(u) & =u^{\circ}, \\
\Phi_{H J}(T) & =r_{H J}^{*}(T), \\
\Gamma_{H J}(T+\operatorname{WAP}(\widehat{H})) & =\widetilde{r}_{H J}^{*}(T) \\
& =r_{H J}^{*}(T)+\operatorname{WAP}(\widehat{J}) \quad(\text { as in Proposition } 4.5),
\end{aligned}
$$


where $u^{\circ}$ denotes the trivial extension of $u$ to $J$ (i.e., $u^{\circ}(x)=0$ if $x \in J \backslash H$ ), and $r_{H J}^{*}$ is the adjoint of the restriction map $r_{H J}: A(J) \rightarrow A(H)$. Also, we define $\Lambda_{H}=\Lambda_{H G}, \Phi_{H}=\Phi_{H G}$, and $\Gamma_{H}=\Gamma_{H G}$.

Lemma 5.2. Let $H$ and $J$ be open subgroups of $G$ such that $H \subseteq J$. Let $\Lambda_{H J}, \Phi_{H J}, \Gamma_{H J}, \Lambda_{H}, \Phi_{H}$, and $\Gamma_{H}$ be the maps from Definition 5.1.

(a) $\Lambda_{H J}$ is an isometric isomorphism from the Banach algebra $A(H)$ onto the Banach subalgebra $A_{H}(J)$ of $A(J)$, where $A_{H}(J)=\{f \in A(J)$ : $\operatorname{supp} f \subseteq H\}$.

(b) $\Phi_{H J}$ is a *-isomorphism (and hence an isometry) from the von Neumann algebra $\mathrm{VN}(H)$ onto the von Neumann subalgebra $\mathrm{VN}_{H}(J)$ of $\mathrm{VN}(J)$.

(c) $\Gamma_{H J}$ is a linear isometry with range $\operatorname{VN}_{H}(J) / \mathrm{WAP}(\widehat{J})$.

(d) If $K$ is an open subgroup of $G$ and $H \subseteq J \subseteq K$, then $\Lambda_{J K} \Lambda_{H J}=$ $\Lambda_{H K}, \Phi_{J K} \Phi_{H J}=\Phi_{H K}$, and $\Gamma_{J K} \Gamma_{H J}=\Gamma_{H K}$. In particular, the maps $\Lambda_{H}$, $\Phi_{H}$, and $\Gamma_{H}$ are compatible with $\Lambda_{H J}, \Phi_{H J}$, and $\Gamma_{H J}$, respectively. That is, $\Lambda_{J} \Lambda_{H J}=\Lambda_{H}, \Phi_{J} \Phi_{H J}=\Phi_{H}$, and $\Gamma_{J} \Gamma_{H J}=\Gamma_{H}$ for all $H \subseteq J$.

Proof. (a) and (b) follow from [5, (3.21)]. (c) holds by Proposition 4.5. And it is easy to check (d) by Definition 5.1.

Summarizing the above discussion, we are ready to give the following decompositions for the Fourier algebra $A(G)$, the von Neumann algebra $\mathrm{VN}(G)$, and the quotient Banach space $\operatorname{VN}(G) / \operatorname{WAP}(\widehat{G})$.

THEOREM 5.3. Let $G$ be a non-discrete locally compact group with $b(G)<$ $d(G)$ and let $G_{0}$ be a $\sigma$-compact open subgroup of $G$. Let $\mathcal{B}=\{B: B \subseteq$ $G$ and $\operatorname{card}(B) \leq b(G)\}$ and let $\mathcal{H}$ be the family of open subgroups of $G$ generated by $G_{0} \cup \bigcup_{g \in B} g G_{0}(B \in \mathcal{B})$. Then:

(1) $\mathcal{H}$ is a directed set under the relation " $\subseteq$ ", $d(H) \leq b(H)$ for all $H \in \mathcal{H}, G=\bigcup_{H \in \mathcal{H}} H$, and $\operatorname{card}(\mathcal{H}) \leq d(G)^{b(G)}$.

(2) For all $H \in \mathcal{H}, A(H)$ is extremely non-Arens regular.

(3) $A(G)=\bigsqcup_{H \in \mathcal{H}} A(H)$ is an inductive union of the Banach algebras $\{A(H)\}_{H \in \mathcal{H}}$ via the isometric isomorphisms $\left\{\Lambda_{H}\right\}_{H \in \mathcal{H}}$.

(4) $\operatorname{VN}(G)=\bigsqcup_{H \in \mathcal{H}} \mathrm{VN}(H)$ is an inductive union of the von Neumann algebras $\{\operatorname{VN}(H)\}_{H \in \mathcal{H}}$ via the $*$-isomorphisms $\left\{\Phi_{H}\right\}_{H \in \mathcal{H}}$.

(5) $\operatorname{VN}(G) / \operatorname{WAP}(\widehat{G})=\bigsqcup_{H \in \mathcal{H}}[\operatorname{VN}(H) / \operatorname{WAP}(\widehat{H})]$ is an inductive union of the quotient Banach spaces $\{\mathrm{VN}(H) / \mathrm{WAP}(\widehat{H})\}_{H \in \mathcal{H}}$ via the linear isometries $\left\{\Gamma_{H}\right\}_{H \in \mathcal{H}}$.

(6) $A(G)=\bigsqcup_{H \in \mathcal{H}} A(H)$ is a WAP-strong inductive union of the algebras $\{A(H)\}_{H \in \mathcal{H}}$.

(7) $\Lambda_{H J}, \Phi_{H J}$, and $\Gamma_{H J}(H, J \in \mathcal{H}$ and $H \subseteq J)$ are the maps compatible with $\left\{\Lambda_{H}\right\}_{H \in \mathcal{H}},\left\{\Phi_{H}\right\}_{H \in \mathcal{H}}$, and $\left\{\Gamma_{H}\right\}_{H \in \mathcal{H}}$ as in Corollary 3.2 and Corollary 3.5 , respectively. 
In particular, if $G$ is metrizable, then $H$ is a $\sigma$-compact open subgroup of $G$ for all $H \in \mathcal{H}$ and $A(G)$ is a WAP-strong inductive union of the separable Fourier algebras $\{A(H)\}_{H \in \mathcal{H}}$.

Proof. Clearly, $\mathcal{H}$ is a directed set under " $\subseteq$ ", $d(H) \leq b(H)$ for all $H \in \mathcal{H}$ (see the second paragraph in this section), and $G=\bigcup_{H \in \mathcal{H}} H$. Let $S$ be a complete set of left coset representatives of $G_{0}$ in $G$ and let $\mathcal{E}=\{B \subseteq S$ : $\operatorname{card}(B) \leq b(G)\}$. It can be seen that $\operatorname{card}(S)=d(G)$ and hence $\operatorname{card}(\mathcal{H}) \leq$ $\operatorname{card}(\mathcal{E}) \leq d(G)^{b(G)}$. Therefore, (1) holds.

(2) and (4) are true according to the discussion in the second paragraph of this section, Lemma 5.2(b), and Definition 3.1.

Note that $A(G) \cap C_{00}(G)$ is norm dense in $A(G)$. So, if $f \in A(G)$, then supp $f$ can be covered by countably many cosets $g G_{0}(g \in G)$. Hence, $\operatorname{supp} T \subseteq H$ for some $H \in \mathcal{H}$. Therefore, $f \in A_{H}(G)=\Lambda_{H}(A(H))$ for some $H \in \mathcal{H}$. By Lemma 5.2(a) and Definition 3.1, (3) holds.

(5) follows from (4) and Lemma 5.2(c).

Let $H \in \mathcal{H}$ and let $r_{H}: A(G) \rightarrow A(H)$ be the restriction map. Then $r_{H} \Lambda_{H}=\operatorname{Id}$ and $\Phi_{H}=r_{H}^{*}$. Thus, $\Lambda_{H}^{*} \Phi_{H}=\Lambda_{H}^{*} r_{H}^{*}=\mathrm{Id}$. It is easy to see that $\Phi_{H}(u \cdot T)=\Lambda_{H}(u) \cdot \Phi_{H}(T)$ for all $u \in A(H)$ and $T \in \mathrm{VN}(H)$ by the fact that $r_{H} \Lambda_{H}=\operatorname{Id}$ and $\Phi_{H}=r_{H}^{*}$. Clearly, $\Phi_{H}(\operatorname{WAP}(\widehat{H}))=\operatorname{WAP}(\widehat{G}) \cap \Phi_{H}(\operatorname{VN}(H))$ and $\Gamma_{H}: \operatorname{VN}(H) / \mathrm{WAP}(\widehat{H}) \rightarrow \operatorname{VN}(G) / \mathrm{WAP}(\widehat{G})$ is the linear isometry lifted by $\Phi_{H}: \mathrm{VN}(H) \rightarrow \mathrm{VN}(G)$. Therefore, $A(G)=\bigsqcup_{H \in \mathcal{H}} A(H)$ is a WAPstrong inductive union of $\{A(H)\}_{H \in \mathcal{H}}$ by (4), (5), and Definition 3.3, i.e., (6) is true.

(7) holds by Lemma $5.2(\mathrm{~d})$ and the uniqueness of the maps $\Lambda_{H J}, \Phi_{H J}$, and $\Gamma_{H J}$ satisfying Corollary 3.2(a) and Corollary 3.5(a), respectively.

Finally, suppose that $G$ is metrizable. Let $H \in \mathcal{H}$. Then $d(H) \leq b(H)=$ $\aleph_{0}$ by (2). Therefore, $H$ is $\sigma$-compact and metrizable and hence $A(H)$ is separable.

Remark 5.4. Let $V$ be any closed $B(G)$-submodule of $\operatorname{VN}(G)$ and let $V_{H}=\Phi_{H}^{-1}\left[V \cap \mathrm{VN}_{H}(G)\right]$. By Remark 4.6, the spaces $\operatorname{WAP}(\widehat{G})$ and $\{\operatorname{WAP}(\widehat{H})\}_{H \in \mathcal{H}}$ in Theorem 5.3(5) can be replaced by $V$ and $\left\{V_{H}\right\}_{H \in \mathcal{H}}$, respectively. Therefore, the inductive union $A(G)=\bigsqcup_{H \in \mathcal{H}} A(H)$ in Theorem 5.3 is more than WAP-strong.

Let $G$ be a locally compact abelian group with the dual group $\Gamma$. Then the Fourier algebra $A(G)$ of $G$ is isometrically isomorphic to the group algebra $L^{1}(\Gamma)$ of $\Gamma$ by the Fourier transform (see Eymard [5, (3.6)]). So, $\operatorname{VN}(G)$ is identified with $L^{\infty}(\Gamma)$. Under these identifications, the module action of $L^{1}(\Gamma)$ on $L^{\infty}(\Gamma)$ is given by

$$
f \cdot \phi=\check{f} * \phi \quad\left(f \in L^{1}(\Gamma) \text { and } \phi \in L^{\infty}(\Gamma)\right),
$$


where $\check{f}(x)=f\left(x^{-1}\right)(x \in \Gamma)$ (see Dunkl and Ramirez [4]). This coincides with the module action of the Banach algebra $L^{1}(\Gamma)$ (taking the convolution as the multiplication) on $L^{\infty}(G)=L^{1}(G)^{*}$. Also, we have $b(G)=d(\Gamma)$ (cf. $[12,(24.48)]$ ) and hence $d(G)=b(\Gamma)$ by the Pontryagin duality theorem. In particular, $G$ is non-discrete if and only if $\Gamma$ is non-compact. Now, for any open subgroup $H$ of $G$, let $N_{H}=\{\gamma \in \Gamma: \gamma(x)=1$ for all $x \in H\}$. Then $\widehat{H} \cong \Gamma / N_{H}$ and $N_{H}(\cong \widehat{G / H})$ is a compact subgroup of $\Gamma$. Applying Theorem 5.3, we obtain the following decomposition for the group algebra of any non-compact locally compact abelian group.

COROLlary 5.5. Let $G$ be a non-compact locally compact abelian group satisfying $d(G)<b(G)$. Then there exists a family $\left\{N_{i}\right\}_{i \in I}$ of compact subgroups of $G$ indexed by a directed set I such that:

(1) $N_{i} \supseteq N_{j} \neq\{e\}$ for all $i, j \in I$ with $i \preceq j$ and $\bigcap_{i \in I} N_{i}=\{e\}$.

(2) $b\left(G / N_{i}\right) \leq d\left(G / N_{i}\right)$ for all $i \in I$ and $\operatorname{card}(I) \leq b(G)^{d(G)}$.

(3) $L^{1}(G)=\bigsqcup_{i \in I} L^{1}\left(G / N_{i}\right)$ is a WAP-strong inductive union via the isometric isomorphisms $\Lambda_{i}: L^{1}\left(G / N_{i}\right) \rightarrow L^{1}(G)$ given by $\Lambda_{i}(f)=f \circ \eta_{i}$ $\left(f \in L^{1}\left(G / N_{i}\right)\right)$, where $\eta_{i}$ is the natural homomorphism of $G$ onto $G / N_{i}$ $(i \in I)$.

REMARK 5.6. Under the assumptions of Theorem 5.3, we also have the inductive union $L^{1}(G)=\bigsqcup_{H \in \mathcal{H}} L^{1}(H)$ of Banach algebras via the isometric isomorphisms $\left\{\Omega_{H}\right\}_{H \in \mathcal{H}}$, where $\Omega_{H}: L^{1}(H) \rightarrow L^{1}(G)$ is defined by $\Omega_{H}(f)=f^{\circ}$ (the trivial extension of $f$ to $G$ ). However, usually $L^{\infty}(G)$ cannot be an inductive union of $\left\{L^{\infty}(H)\right\}_{H \in \mathcal{H}}$. For example, suppose that $d(G)=2^{\alpha}$ for some $\alpha \geq b(G)$. Note that $\operatorname{card}(\mathcal{H}) \leq d(G)^{b(G)}=2^{\alpha}$ and $D\left(L^{1}(H)\right) \leq b(H)=b(G)$ for all $H \in \mathcal{H}$, where $D\left(L^{1}(H)\right)$ is the smallest cardinality of a norm dense subset of $L^{1}(H)$. It follows that $\operatorname{card}\left(\bigcup_{H \in \mathcal{H}} L^{\infty}(H)\right) \leq 2^{b(G)} \operatorname{card}(\mathcal{H}) \leq 2^{\alpha}=d(G)<2^{d(G)} \leq \operatorname{card}\left(L^{\infty}(G)\right)$, i.e., $\operatorname{card}\left(\bigcup_{H \in \mathcal{H}} L^{\infty}(H)\right)<\operatorname{card}\left(L^{\infty}(G)\right)$. Therefore, the inductive union $L^{1}(G)=\bigsqcup_{H \in \mathcal{H}} L^{1}(H)$ is not WAP-strong.

According to Theorem 5.3(2), for each $H \in \mathcal{H}$, there exists a closed linear subspace $Z_{H}$ of $\operatorname{VN}(H) / \mathrm{WAP}(\widehat{H})$ and a continuous linear map $\Pi_{H}: Z_{H} \rightarrow$ $\mathrm{VN}(H)$ such that $\Pi_{H}\left(Z_{H}\right)=\mathrm{VN}(H)$. We will consider whether the family $\left\{\left\{Z_{H}, \Pi_{H}\right\}: H \in \mathcal{H}\right\}$ is compatible with the maps $\Phi_{H J}$ and $\Gamma_{H J}(H, J \in \mathcal{H}$ and $H \subseteq J)$. For this purpose, we will need the following two lemmas.

Lemma 5.7. Let $H$ and $J$ be open subgroups of $G$ with $H \subseteq J$ and let $\Lambda_{H J}, \Phi_{H J}$, and $\Gamma_{H J}$ be the maps defined in Definition 5.1. Let $\Psi_{H J}=\Lambda_{H J}^{*}$. Then:

(a) $\Psi_{H J}: \mathrm{VN}(J) \rightarrow \mathrm{VN}(H)$ is a continuous linear surjection, $\left\|\Psi_{H J}\right\|$ $=1$, and $\Psi_{H J} \Phi_{H J}=\mathrm{Id}$. 
(b) $\Psi_{H J}(\operatorname{WAP}(\widehat{J}))=\operatorname{WAP}(\widehat{H})$.

Define $\Theta_{H J}: \operatorname{VN}(J) / \mathrm{WAP}(\widehat{J}) \rightarrow \operatorname{VN}(H) / \mathrm{WAP}(\widehat{H})$ by $\Theta_{H J}(T+\mathrm{WAP}(\widehat{J}))$ $=\Psi_{H J}(T)+\operatorname{WAP}(\widehat{H})(T \in V N(J))$. Then:

(c) $\Theta_{H J}$ is a continuous linear surjection, $\left\|\Theta_{H J}\right\|=1$, and $\Theta_{H J} \Gamma_{H J}=\mathrm{Id}$.

(d) If $K$ is an open subgroup of $G$ and $H \subseteq J \subseteq K$, then $\Psi_{H J} \Psi_{J K}=$ $\Psi_{H K}$ and $\Theta_{H J} \Theta_{J K}=\Theta_{H K}$.

Proof. (a) This follows from [5, (3.21)].

(b) Note that $\Psi_{H J} \Phi_{H J}=\operatorname{Id}$ and $\Phi_{H J}(\operatorname{WAP}(\widehat{H})) \subseteq \operatorname{WAP}(\widehat{J})$ (see $[2$, Lemma 4.2]). So, $\operatorname{WAP}(\widehat{H}) \subseteq \Psi_{H J}(\operatorname{WAP}(\widehat{J}))$. On the other hand, for $u \in$ $A(H)$ and $T \in \operatorname{VN}(J)$, we have $u \cdot \Psi_{H J}(T)=\Psi_{H J}\left(\Lambda_{H J}(u) \cdot T\right)$. Therefore, $\Psi_{H J}(\operatorname{WAP}(\widehat{J})) \subseteq \operatorname{WAP}(\widehat{H})$ and hence $\Psi_{H J}(\operatorname{WAP}(\widehat{J}))=\operatorname{WAP}(\widehat{H})$.

(c) By (a) and (b), $\Theta_{H J}$ is well-defined, linear, continuous, and onto. And $\Theta_{H J} \Gamma_{H J}=\mathrm{Id}$ since $\Psi_{H J} \Phi_{H J}=\mathrm{Id}$. Note that $\Gamma_{H J}$ is an isometry. So we have $\left\|\Theta_{H J}\right\| \geq 1$. On the other hand, by the definition of $\Theta_{H J}$ and by the fact that $\left\|\Psi_{H J}\right\|=1$, we get $\left\|\Theta_{H J}\right\| \leq 1$. Therefore, $\left\|\Theta_{H J}\right\|=1$.

(d) Since $\Lambda_{J K} \Lambda_{H J}=\Lambda_{H K}$, by taking the adjoint, we have $\Psi_{H J} \Psi_{J K}=$ $\Psi_{H K}$ and hence $\Theta_{H J} \Theta_{J K}=\Theta_{H K}$.

Remark 5.8. Comparing to the diagram in Proposition 4.5, we now have the following commutative diagram:

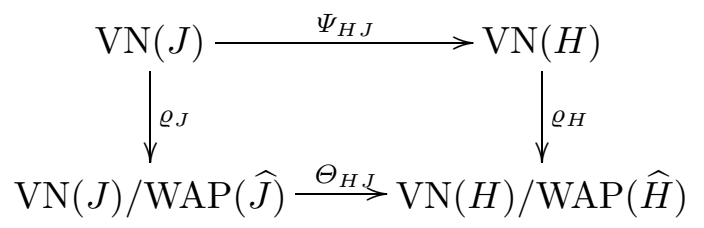

where $\varrho_{H}$ and $\varrho_{J}$ are the canonical quotient maps.

Lemma 5.9. Let $G, G_{0}$, and $\mathcal{H}$ be as in Theorem 5.3. Let $\mu$ be the initial ordinal with $|\mu|=b\left(G_{0}\right)(=b(G))$ and $X=\{\alpha: \alpha<\mu\}$. Then there exists a continuous linear surjection $\omega_{H}: \operatorname{VN}(H) / \mathrm{WAP}(\widehat{H}) \rightarrow l^{\infty}(X)$ for each $H \in \mathcal{H}$ such that the family $\left\{\left\|\omega_{H}\right\|: H \in \mathcal{H}\right\}$ is bounded by a constant which depends only on $b(G)$.

Furthermore, if $H, J \in \mathcal{H}$ and $H \subseteq J$, then $\omega_{H} \Theta_{H J}=\omega_{J}$ and we have the following commutative diagram:

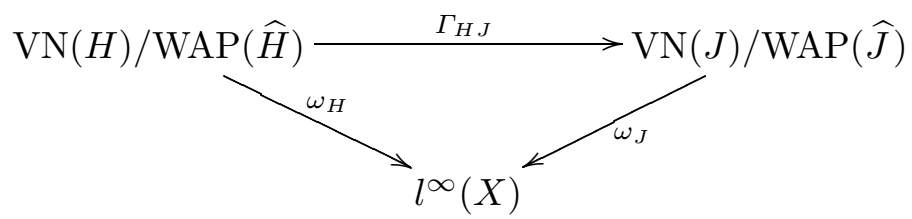

Proof. Let $\pi: \operatorname{VN}\left(G_{0}\right) \rightarrow l^{\infty}(X)$ be the map constructed in $\mathrm{Hu}[9$, Theorem 5.1]. According to [9, Theorem 5.1] and its proof, $\pi$ is a continuous 
linear surjection, $\|\pi\|=1$, and $\pi\left(\operatorname{WAP}\left(\widehat{G}_{0}\right)\right) \subseteq c(X)$, where $c(X)=\{f \in$ $l^{\infty}(X): \lim _{\alpha} f(\alpha)$ exists $\}$. Note that $l^{\infty}(X) / c(X)$ contains an isomorphic copy of $l^{\infty}(X)$ (see [9, Lemma 3.2]) and $l^{\infty}(X)$ is an injective Banach space (see [14]). So, there exists a continuous linear surjection $\tau: l^{\infty}(X) / c(X) \rightarrow$ $l^{\infty}(X)$. Define $\omega: \operatorname{VN}\left(G_{0}\right) / \operatorname{WAP}\left(\widehat{G}_{0}\right) \rightarrow l^{\infty}(X)$ by $\omega\left(T+\operatorname{WAP}\left(\widehat{G}_{0}\right)\right)=$ $\tau(\pi(T)+c(X))\left(T \in \operatorname{VN}\left(G_{0}\right)\right)$. Then $\omega$ is well defined, linear, continuous, onto $l^{\infty}(X)$, and $\|\omega\| \leq\|\tau\|$.

For $H \in \mathcal{H}$, let $\omega_{H}=\omega \Theta_{G_{0} H}$, where $\Theta_{G_{0} H}: \operatorname{VN}(H) / \operatorname{WAP}(\widehat{H}) \rightarrow$ $\operatorname{VN}\left(G_{0}\right) / \operatorname{WAP}\left(\widehat{G}_{0}\right)$ is the surjection as defined in Lemma 5.7. Then $\omega_{H}$ is continuous, linear, onto $l^{\infty}(X)$, and $\left\|\omega_{H}\right\|=\left\|\omega \Theta_{G_{0} H}\right\| \leq\|\omega\| \leq\|\tau\|$. It turns out that the family $\left\{\left\|\omega_{H}\right\|: H \in \mathcal{H}\right\}$ is bounded by the constant $\|\tau\|$ which depends only on $\operatorname{card}(X)=b(G)$.

Suppose $H, J \in \mathcal{H}$ and $H \subseteq J$. Then $\Theta_{G_{0} H} \Theta_{H J}=\Theta_{G_{0} J}$ (Lemma 5.7(d)). Thus, $\omega \Theta_{G_{0} H} \Theta_{H J}=\omega \Theta_{G_{0} J}$, i.e., $\omega_{H} \Theta_{H J}=\omega_{J}$. But $\Theta_{H J} \Gamma_{H J}=\operatorname{Id}$ (Lemma 5.7(c)). It follows that $\omega_{H}=\omega_{J} \Gamma_{H J}$ and hence the diagram commutes.

Now we have the following inductive compatibility of the pairs $\left\{Z_{H}, \Pi_{H}\right\}$ $(H \in \mathcal{H})$ with the maps $\Phi_{H J}$ and $\Gamma_{H J}$.

THEOREM 5.10. The following hold under the assumptions of Theorem 5.3:

(1) For each $H \in \mathcal{H}$, there exists a closed linear subspace $Z_{H}$ of the quotient $\mathrm{VN}(H) / \mathrm{WAP}(\widehat{H})$ and a continuous linear map $\Pi_{H}: Z_{H} \rightarrow \mathrm{VN}(H)$ such that $\Pi_{H}\left(Z_{H}\right)=\mathrm{VN}(H)$.

(2) There exists a constant $M>0$ (which depends only on $b(G))$ such that $\left\|\Pi_{H}\right\| \leq M$ for all $H \in \mathcal{H}$.

(3) Let $K \in \mathcal{H}$ and let $\mathcal{H}_{K}=\{H \subseteq \mathcal{H}: H \subseteq K\}$. Then, for each $H \in \mathcal{H}_{K}$, a pair $\left\{Z_{H}, \Pi_{H}\right\}$ as in (1) can be chosen such that the family $\left\{\left\{Z_{H}, \Pi_{H}\right\}: H \in \mathcal{H}_{K}\right\}$ is compatible with the maps $\Phi_{H J}$ and $\Gamma_{H J}(H, J \in$ $\mathcal{H}_{K}$ and $\left.H \subseteq J\right)$. That is, if $H, J \in \mathcal{H}_{K}$ and $H \subseteq J$, then $\Gamma_{H J}\left(Z_{H}\right) \subseteq Z_{J}$ and the following diagram commutes when $\Gamma_{H J}$ is restricted to $Z_{H}$ :

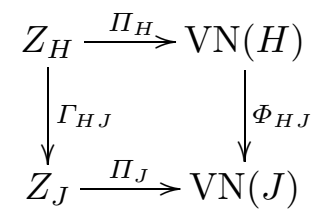

Proof. (1) This follows from Theorem 5.3(2).

(2) Let $H \in \mathcal{H}$. Then $d(H) \leq b(H)$ (Theorem 5.3(1)) and hence $\mathcal{D}(A(H))$ $=b(H)=b(G)=\operatorname{card}(X)$, where $\mathcal{D}(A(H))$ is the smallest cardinality of a norm dense subset of $A(H)$ and $X$ is the same set as in Lemma 5.9. Let $\left\{u_{\alpha}: \alpha \in X\right\}$ be a norm dense subset of the unit ball in $A(H)$ and let 
$t_{H}: \mathrm{VN}(H) \rightarrow l^{\infty}(X)$ be defined by $t_{H}(T)(\alpha)=\left\langle T, u_{\alpha}\right\rangle(T \in \mathrm{VN}(H)$ and $\alpha \in X)$. Then $t_{H}$ is a linear isometry. Let $\omega_{H}: \operatorname{VN}(H) / \operatorname{WAP}(\widehat{H}) \rightarrow$ $l^{\infty}(X)$ be the surjection as constructed in Lemma 5.9. We take $Z_{H}=$ $\omega_{H}^{-1}\left[t_{H}(\mathrm{VN}(H))\right](\subseteq \mathrm{VN}(H) / \mathrm{WAP}(\widehat{H}))$ and $\Pi_{H}=t_{H}^{-1}\left(\left.\omega_{H}\right|_{Z_{H}}\right)$. Then $Z_{H}$ is a closed linear subspace of $\operatorname{VN}(H) / \mathrm{WAP}(\widehat{H}), \Pi_{H}: Z_{H} \rightarrow \mathrm{VN}(H)$ is a continuous linear map, and $\Pi_{H}\left(Z_{H}\right)=\mathrm{VN}(H)$.

It is clear that $\left\|\Pi_{H}\right\| \leq\left\|\omega_{H}\right\|$. By Lemma 5.9 , the family $\left\{\left\|\Pi_{H}\right\|: H \in\right.$ $\mathcal{H}\}$ is bounded by a constant which depends only on $b(G)$.

(3) Let $K \in \mathcal{H}$. Let $t_{K}, Z_{K}$, and $\Pi_{K}$ be as constructed in (2). Let $H \in \mathcal{H}_{K}$ and $t_{H}^{\prime}=t_{K} \Phi_{H K}$. Then $t_{H}^{\prime}: \operatorname{VN}(H) \rightarrow l^{\infty}(X)$ is also a linear isometry since $\Phi_{H K}$ is an isometry. Now we take $Z_{H}=\omega_{H}^{-1}\left[t_{H}^{\prime}(\operatorname{VN}(H))\right](\subseteq$ $\operatorname{VN}(H) / \operatorname{WAP}(\widehat{H}))$ and $\Pi_{H}=\left(t_{H}^{\prime}\right)^{-1}\left(\left.\omega_{H}\right|_{Z_{H}}\right)$. Then $\Pi_{H}: Z_{H} \rightarrow \operatorname{VN}(H)$ is also a continuous linear surjection and we still have $\left\|\Pi_{H}\right\| \leq\left\|\omega_{H}\right\|$.

Suppose that $H, J \in \mathcal{H}_{K}$ and $H \subseteq J$. Since $\Phi_{H K}=\Phi_{J K} \Phi_{H J}$, we have $\Phi_{H K}(\mathrm{VN}(H))=\Phi_{J K}\left[\Phi_{H J}(\operatorname{VN}(H))\right] \subseteq \Phi_{J K}(\operatorname{VN}(J))$ and hence $t_{K} \Phi_{H K}(\mathrm{VN}(H)) \subseteq t_{K} \Phi_{J K}(\mathrm{VN}(J))$, i.e., $t_{H}^{\prime}(\mathrm{VN}(H)) \subseteq t_{J}^{\prime}(\mathrm{VN}(J))$. Note that $\omega_{J} \Gamma_{H J}=\omega_{H}$ (Lemma 5.9). Therefore, we have

$$
\Gamma_{H J}\left[\omega_{H}^{-1}\left(t_{H}^{\prime}(\mathrm{VN}(H))\right)\right] \subseteq \omega_{J}^{-1}\left(t_{H}^{\prime}(\mathrm{VN}(H))\right) \subseteq \omega_{J}^{-1}\left[t_{J}^{\prime}(\mathrm{VN}(J))\right],
$$

i.e., $\Gamma_{H J}\left(Z_{H}\right) \subseteq Z_{J}$. Finally, the construction of $\left\{Z_{H}, \Pi_{H}\right\}$ and $\left\{Z_{J}, \Pi_{J}\right\}$ makes the diagram commutative.

RemarK 5.11. Let $K \in \mathcal{H}$ and $\left\{Z_{K}, \Pi_{K}\right\}$ be the same as constructed in Theorem 5.10(2). If $H, J \in \mathcal{H}_{K}$ with $H \subseteq J$ and $\left\{Z_{H}, \Pi_{H}\right\},\left\{Z_{J}, \Pi_{J}\right\}$ are chosen as in the proof of Theorem 5.10(3), then we only have $Z_{H} \subseteq$ $\Theta_{H J}\left(Z_{J}\right)$, where $\Theta_{H J}: \operatorname{VN}(J) / \operatorname{WAP}(\widehat{J}) \rightarrow \operatorname{VN}(H) / \operatorname{WAP}(\widehat{H})$ is the surjection as defined in Lemma 5.7. So, generally, we cannot simultaneously have the following commutative diagram when $\Theta_{H J}$ is restricted to $Z_{J}$ :

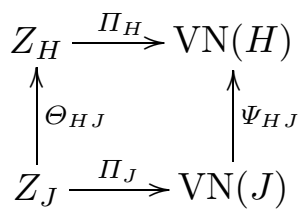

However, for $H \in \mathcal{H}_{K}$, if we let $Q_{H}=\Theta_{H K}\left(Z_{K}\right)(\subseteq \operatorname{VN}(H) / \operatorname{WAP}(\widehat{H}))$ and let $\Sigma_{H}: Q_{H} \rightarrow \mathrm{VN}(H)$ be defined by

$$
\Sigma_{H}\left[\Theta_{H K}(T+\operatorname{WAP}(\widehat{K}))\right]=\Psi_{H K} \Pi_{K}(T+\operatorname{WAP}(\widehat{K}))\left(T+\operatorname{WAP}(\widehat{K}) \in Z_{K}\right),
$$

then it can be seen that $Q_{H}$ is a linear subspace of $\operatorname{VN}(H) / \mathrm{WAP}(\widehat{H}), \Sigma_{H}$ : $Q_{H} \rightarrow \mathrm{VN}(H)$ is well defined, linear, onto $\mathrm{VN}(H)$, and $\left\|\Sigma_{H}\right\| \leq\|\tau\|$, where $\tau: l^{\infty}(X) / c(X) \rightarrow l^{\infty}(X)$ is the surjection as appeared in the proof of Lemma 5.9. Let $Y_{H}$ denote the norm closure of $Q_{H}$ in $\operatorname{VN}(H) / \operatorname{WAP}(\widehat{H})$ and extend $\Sigma_{H}$ continuously to $Y_{H}$. Then $\Sigma_{H}: Y_{H} \rightarrow \mathrm{VN}(H)$ is a continuous 
linear surjection. Now, if $H, J \in \mathcal{H}_{K}$ and $H \subseteq J$, then $\Theta_{H J}\left(Q_{J}\right) \subseteq Q_{H}$ and hence $\Theta_{H J}\left(Y_{J}\right) \subseteq Y_{H}$. Also, we have $\Sigma_{H} \Theta_{H J}\left[\Theta_{J K}(T+\operatorname{WAP}(\widehat{K}))\right]=$ $\Psi_{H J} \Sigma_{J}\left[\Theta_{J K}(T+\operatorname{WAP}(\widehat{K}))\right]$ for $\Theta_{J K}(T+\operatorname{WAP}(\widehat{K})) \in Q_{J}$ and thus the following diagram commutes when $\Theta_{H J}$ is restricted to $Y_{J}$ :

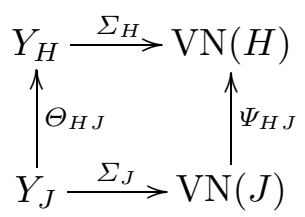

But, in this case, we do not have $\Gamma_{H J}\left(Y_{H}\right) \subseteq Y_{J}$ and hence we cannot have $\left.\Sigma_{J} \Gamma_{H J}\right|_{Y_{H}}=\Phi_{H J} \Sigma_{H}$, i.e., we do not have the commutative diagram in Theorem 5.10 when $\left\{Z_{H}, \Pi_{H}\right\}$ and $\left\{Z_{J}, \Pi_{J}\right\}$ are replaced by $\left\{Y_{H}, \Sigma_{H}\right\}$ and $\left\{Y_{J}, \Sigma_{J}\right\}$, respectively.

It is not clear whether in Theorem 5.10 we could choose a family $\left\{\left\{Z_{H}, \Pi_{H}\right\}: H \in \mathcal{H}\right\}$ compatible with all of the maps $\Phi_{H J}$ and $\Gamma_{H J}$ $(H, J \in \mathcal{H}$ and $H \subseteq J)$. If so, then we would be able to obtain a continuous linear surjection $\Pi: \bigcup_{H \in \mathcal{H}} \Gamma_{H}\left(Z_{H}\right) \rightarrow \mathrm{VN}(G)$ and hence we would be able to conclude that $A(G)$ is extremely non-Arens regular. For this reason, we give the following version of extreme non-Arens regularity.

Definition 5.12. Let $A$ be a Banach algebra. $A$ is called inductively extremely non-Arens regular if there exists a family $\left\{A_{i}\right\}_{i \in I}$ of Banach algebras such that:

(1) For each $i \in I, A_{i}$ is extremely non-Arens regular.

(2) $A=\bigsqcup_{i \in I} A_{i}$ is a WAP-strong inductive union of $\left\{A_{i}\right\}_{i \in I}$ with $A^{*}=$ $\bigsqcup_{i \in I} A_{i}^{*}$ via $\left\{\Phi_{i}\right\}_{i \in I}$ and $A^{*} / \operatorname{WAP}(A)=\bigsqcup_{i \in I}\left[A_{i}^{*} / \operatorname{WAP}\left(A_{i}\right)\right]$ via $\left\{\Gamma_{i}\right\}_{i \in I}$.

(3) Let $k \in I$ and let $I_{k}=\{i \in I: i \preceq k\}$. Then, for each $i \in I_{k}$, there exists a closed linear subspace $Z_{i}$ of $A_{i}^{*} / \mathrm{WAP}\left(A_{i}\right)$ and a continuous linear surjection $\Pi_{i}: Z_{i} \rightarrow A_{i}^{*}$ such that $\left\{\left\|\Pi_{i}\right\|: i \in I_{k}\right\}$ is bounded (by a constant independent of $k$ ) and $\left\{\left\{Z_{i}, \Pi_{i}\right\}: i \in I_{k}\right\}$ is compatible. That is, if $i, j \in I_{k}$ and $i \preceq j$, then $\Gamma_{i j}\left(Z_{i}\right) \subseteq Z_{j}$ and $\Phi_{i j} \Pi_{i}=\left.\Pi_{j} \Gamma_{i j}\right|_{Z_{i}}$, where $\Phi_{i j}$ and $\Gamma_{i j}$ are the same maps as in Corollary 3.5.

Combining Theorem 5.3 and Theorem 5.10 with [10, Corollary 4.2 and Remark 4.7], we are able to deduce the non-Arens regularity of $A(G)$ as follows.

COROLlary 5.13. Let $G$ be a non-discrete locally compact group. Then:

(1) $A(G)$ is extremely non-Arens regular if $b(G) \geq d(G)$.

(2) $A(G)$ is inductively extremely non-Arens regular if $b(G)<d(G)$. 
As an immediate consequence of Corollary 5.13, we have the following result on the non-Arens regularity of the group algebra $L^{1}(G)$ of any noncompact locally compact abelian group $G$.

Corollary 5.14. Let $G$ be a non-compact locally compact abelian group. Then:

(1) $L^{1}(G)$ is extremely non-Arens regular if $b(G) \leq d(G)$.

(2) $L^{1}(G)$ is inductively extremely non-Arens regular if $b(G)>d(G)$.

Acknowledgments. This paper was partly written while the author was visiting Prof. B. Forrest at the University of Waterloo in Fall 2000. She is indebted to Prof. B. Forrest and the Department of Pure Mathematics at the University of Waterloo for the hospitality and support.

We thank the referee and the editor for the valuable suggestions.

\section{References}

[1] R. Arens, The adjoint of a bilinear operation, Proc. Amer. Math. Soc. 2 (1951), 839-848.

[2] C. Chou, Almost periodic operators in $\mathrm{VN}(G)$, Trans. Amer. Math. Soc. 317 (1990), 229-253.

[3] J. Duncan and S. A. R. Hosseinium, The second dual of a Banach algebra, Proc. Roy. Soc. Edinburgh Sect. A 84 (1979), 309-325.

[4] C. F. Dunkl and D. E. Ramirez, Weakly almost periodic functionals on the Fourier algebra, Trans. Amer. Math. Soc. 185 (1973), 501-514.

[5] P. Eymard, L'algèbre de Fourier d'un groupe localement compact, Bull. Soc. Math. France 92 (1964), 181-236.

[6] B. Forrest, Arens regularity and discrete groups, Pacific J. Math. 151 (1991), 217227.

[7] E. E. Granirer, Day points for quotients of the Fourier algebra $A(G)$, extreme nonergodicity of their duals and extreme non-Arens regularity, Illinois J. Math. 40 (1996), 402-419.

[8] C. Herz, Harmonic synthesis for subgroups, Ann. Inst. Fourier (Grenoble) 23 (1973), 91-123.

[9] Z. Hu, The von Neumann algebra $\mathrm{VN}(G)$ of a locally compact group and quotients of its subspaces, Canad. J. Math. 49 (1997), 1117-1138.

[10] - Extreme non-Arens regularity of quotients of the Fourier algebra $A(G)$, Colloq. Math. 72 (1997), 237-249.

[11] -, Open subgroups of $G$ and almost periodic functionals on $A(G)$, Proc. Amer. Math. Soc. 128 (2000), 2473-2478.

[12] E. Hewitt and K. A. Ross, Abstract Harmonic Analysis I, Springer, New York, 1979.

[13] A. T. Lau, The second conjugate of the Fourier algebra of a locally compact group, Trans. Amer. Math. Soc. 267 (1981), 53-63.

[14] J. Lindenstrauss and L. Tzafriri, Classical Banach Spaces, Vol. I, Springer, New York, 1977. 
[15] J. S. Pym, The convolution of functionals on spaces of bounded functions, Proc. London Math. Soc. 15 (1965), 84-104.

Department of Mathematics and Statistics

University of Windsor

Windsor, Ontario, Canada N9B 3P4

E-mail: zhiguohu@uwindsor.ca

Received March 5, 2001

Revised version December 20, 2001 